\title{
Respuesta del Tumor Venéreo Transmisible Canino a Presentaciones de Vincristina de Patente y Genérica
}

\author{
Response of Canine Transmissible Venereal Tumor to Various Vincristine \\ Presentations
}
Susana Miguel De la $\mathrm{Cruz}^{3}$, Israel A. Quijano-Hernández ${ }^{1,4}$, Javier Del Ángel- Caraza $^{1}$, José S. Martínez Castañeda ${ }^{2}$, José M. Victoria-Mora ${ }^{1}$, Marco A. Barbosa-Mireles ${ }^{1}$

\section{Resumen}

El objetivo del presente estudio fue comparar la respuesta de perros infectados naturalmente con el Tumor Venéreo Transmisible (TVTc) al tratamiento con vincristina comercial de patente y genérica. Se trabajó con 12 perros infectados naturalmente y con diagnóstico por citología y PCR. Los perros fueron asignados aleatoriamente a un tratamiento semanal con $0.025 \mathrm{mg} / \mathrm{kg}$ de vincristina de patente comercial o de tipo genérico, hasta que dos citologías consecutivas resultaran negativas. Se hicieron pruebas de citología y hemograma antes de cada aplicación para determinar reacciones adversas al tratamiento. En ambos grupos se aplicó un máximo de cinco dosis para dar por concluido el tratamiento. El mínimo de aplicaciones en el grupo Patente fue de cuatro, mientras que en el grupo Genérico fue de tres aplicaciones, aunque sin diferencia significativa en el número de dosis aplicadas ni en el tiempo de remisión. Los perros de ambos grupos presentaron algún tipo de reacción adversa ligera sin mostrar diferencia estadística entre grupos. En ningún caso hubo que descontinuar el tratamiento por este tipo de reacciones. Se concluye que las presentaciones de patente y genérica son igualmente eficaces para el tratamiento de TVTc.

Palabras clave: toxicidad, quimioterapéuticos, perro, eficacia-seguridad

\section{Abstract}

The aim of this study was to compare the response of naturally infected dogs with transmissible venereal tumor (TVT) to treatment with two sources of vincristine: patented and generic. Twelve naturally infected dogs diagnosed with TVT by cytology and PCR

\footnotetext{
${ }^{1}$ Hospital Veterinario para Pequeñas Especies, ${ }^{2}$ Centro de Investigación y Estudios Avanzados en Salud Animal, ${ }^{3}$ Facultad de Medicina Veterinaria y Zootecnia, Universidad Autónoma del Estado de México (FMVZ-UAEM). Toluca, México

${ }^{4}$ E-mail: iaquijanoh@uaemex.mx
}

Recibido: 4 de marzo de 2015

Aceptado para publicación: 23 de junio de 2015 
were randomly assigned to a weekly treatment with vincristine at a dose of $0.025 \mathrm{mg} / \mathrm{kg}$ until two consecutive cytological evaluations resulted negative. Cytology tests and haemograms were performed prior to vincristine applications to evaluate adverse reaction to treatment. In both groups, a maximum of five applications were applied to obtain a successful treatment. The minimum number of applications in the Patent group was four while in the Generic group was three, although without significant difference between groups in number of applications or time for healing. Dogs in both groups showed some kind of adverse reactions to the treatment but without statistical difference between groups. There was no need to discontinue the treatment in any case. It is concluded that the patent and generic presentations of vincristine are equally effective for treating TVT in dogs.

Key words: toxicity, chemotherapeutics, dog, efficacy-safety

\section{INTRODUCCIÓN}

El tumor venéreo transmisible canino (TVTc), conocido también como sarcoma infeccioso, linfosarcoma venéreo, granuloma venéreo, condiloma canino y sarcoma de «Sticker», es una neoplasia específica del perro (Ortega et al., 2003). Tiene una incidencia de 6 a 30 casos por cada 100000 perros. Su crecimiento es local y la tasa de metástasis es de 1 a 6\% (Strakova y Murchison, 2014).

El origen celular del TVT se presume de células redondas no diferenciadas de origen reticuloendotelial histiocítico. Aun cuando se ha especulado un origen viral, estos tumores no pueden ser transmitidos por filtrados libres de células (Salamanca et al., 2008). Las células del TVTc se caracterizan por presentar un menor número de cromosomas que las células normales del perro y por tener inserto una secuencia genética conocida como 'long interspersed nuclear element' cerca del gene c-myc (Liao et al., 2003). Esta clona de células ha podido ser transmitida entre las poblaciones caninas, actuando más como una célula parásito que como una neoplasia in stricto sensu (VonHoldt y Ostrander, 2006).

El TVTc se suele asociar al tracto genital (vagina y pene) pero, en realidad, su desarrollo es el resultado del trasplante de célu- las tumorales en un sitio susceptible, de preferencia en superficies mucosas con abrasiones, mucosa nasal, bucal, conjuntiva y región anal, aunque también se puede desarrollar en una herida cutánea. Por este motivo, según su localización, se le clasifica en genital y extragenital (Mukaratirwa y Gruys, 2003).

Es una patología altamente contagiosa entre los perros, ya que puede ser transmitida durante el coito, mordeduras, olfateo y por el lamido de laceraciones. El TVTc es el primer tumor transmitido experimentalmente, logrado por el veterinario ruso Nowinsky en 1876 (Hsiao et al., 2002). Tiene una distribución global, especialmente en países tropicales y subtropicales, y en perros callejeros sexualmente activos (Ortega et al., 2003). No hay discriminación por edad o sexo; sin embargo, los perros mayores de un año tienen más riesgos en áreas endémicas (Das y Kumar, 2000). Se transmite naturalmente como un aloinjerto, directamente de un perro a otro (Murgía et al., 2006).

El tratamiento con sulfato de vincristina en dosis semanal de $0.025 \mathrm{mg} / \mathrm{kg}$ tiene buenos resultados y los efectos colaterales como vómito, diarrea, anorexia y neutropenia son mínimos (Vermooten, 1987). Habitualmente se utiliza una presentación comercial que contiene $1.0 \mathrm{mg}$ de sulfato de vincristina, pudiéndose requerir entre 4 y 7 aplicaciones, conforme a la evolución del paciente (Brown $e t$ 


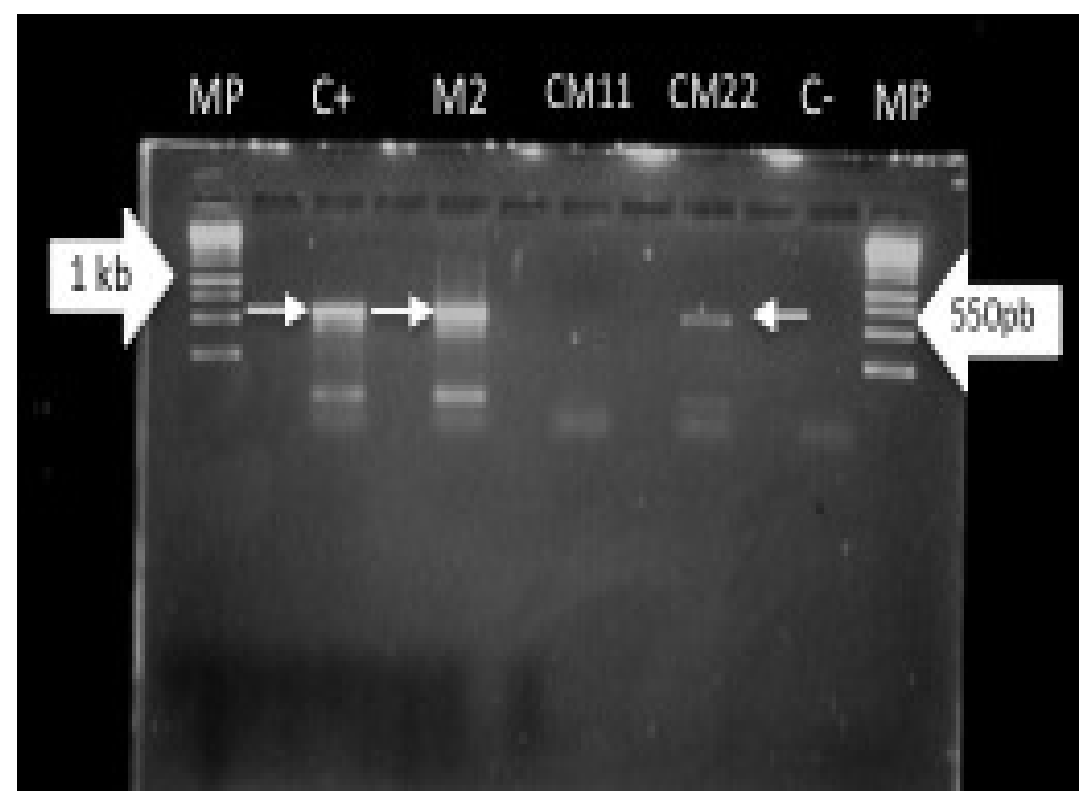

Figura 1. Evaluación por PCR de dos pacientes positivos y uno negativo a tumor venéreo transmisible canino (TVTc). Gel de agarosa mostrando el fragmento amplificado por PCR de $550 \mathrm{pb}$ para TVT, originado por el rearreglo LINE/c-myc; Carri 1, MP: $1 \mathrm{~kb}$; carril 3, C+ o control positivo; carril 5, M2 o perro G2; carril 7, perro CM11; carril 9, CM22 o P3; carril 11, C- o control negativo). Flecha izquierda 1kb, flecha derecha $550 \mathrm{pb}$

al., 1980; Tomiyasu et al., 2011). No obstante, se ha demostrado que la vincristina causa alteraciones gastrointestinales, aún más que la doxorrubicina, en el perro (Tomiyasu et al., 2011). De hecho, la aplicación de vincristina incrementa el riesgo de desarrollo de sepsis cuando se administran más de nueve dosis (Sorenmo et al., 2010). Además, altera la agregación plaquetaria (Grau-Bassas et al., 2000).

Las presentaciones comerciales genéricas tienen un costo reducido comparado al producto comercial de patente; sin embargo, ante la incertidumbre del funcionamiento de ciertos medicamentos genéricos y la aparente falta de efectividad y aumento de toxicosis, el grueso de los veterinarios siguen utilizando la presentación de patente con la cual han tenido mayor experiencia. Con base en esto, el objetivo del presente estudio fue comparar la respuesta de perros infectados naturalmente con TVTc y tratados con preparaciones de vincristina de patente y genérica.

\section{Materiales y Métodos}

\section{Animales, Diagnóstico y Tratamiento}

Se trabajó con 13 perros aparentemente infectados con TVTc, recibidos en el Hospital Veterinario para Pequeñas Especies de la Facultad de Medicina Veterinaria y Zootecnia, Universidad Autónoma del Estado de México (FMVZ-UAEM). Estos fueron 3 hembras y 10 machos adultos jóvenes de 1 a 10 años, de razas medianas (Cobrador de Labrador, Labrador Dorado, Doberman, Dálmata, Fila Brasileiro, Cocker Spaniel y Pastor Alemán), así como dos perros sin raza definida pero de tamaño grande (mayores de 27-30 kg). Los perros fueron asignados aleatoriamente a dos grupos de tratamiento: vincristina de patente y vincristina genérica, de disponibilidad en el mercado local.

Se realizó un estudio citológico por medio de punción con aguja delgada o hisopado- 
para realizar el diagnóstico de TVTc, utilizando colorante de tipo Romanowsky (ErünalMaral et al., 2000; Marcos et al., 2006). Además, se obtuvo tejido del tumor conservado en solución RNAlater (Ambion, Life Technologies, EEUU) y refrigerado para la extracción de ADN con el kit Genomic DNA Purification (Fermentas Inc., Canadá).

Se utilizó la técnica del PCR para confirmar que el tumor proviene de TVTc. Para esto, se amplificó la inserción de LINE en el gen $c-M y c$ en su región conservada empleando los iniciadores MycS-2: 5'ATTCCTACGAATGAATGATTGGCCAGA3' y LINE AS-1: 5'AGACACATAGA-TCAGTGGAACAGAAT3'. La PCR se realizó en el Mastercycler personal (Eppendorf $®)$. En un volumen final de 50 ìl se colocó el DNA purificado con $1.5 \mathrm{nM}$ de $\mathrm{MgCl}_{2}$, buffer green al $1 \mathrm{X}, 1 \mathrm{U}$ de Flexi taq DNA polymerasa (Promega), 10 pmol de cada dNTP's (Promega) y 10 pmol de cada uno de los iniciadores. La solución fue homogenizada con agua libre de nucleasas (Fermentas), según ha sido descrito por Liao et al. (2003) y Bautista et al. (2011), obteniendo un fragmento de 550 pares de bases. La presencia del fragmento se evidenció en gel de agarosa al $1 \%$ a $80 \mathrm{~V}$ por $90 \mathrm{~min}$ (Fig. 1). Doce perros dieron positivo, de modo que el perro que resultó negativo fue excluido del estudio.

Luego del diagnóstico y antes del tratamiento se realizaron estudios de funcionamiento orgánico rutinarios (renal, hepático, hemograma, urianálisis) para determinar la factibilidad de recibir quimioterapia. Los perros de cada grupo recibieron $0.025 \mathrm{mg} / \mathrm{kg}$ de sulfato de vincristina de patente (Oncovin, Sandoz-México) o genérica (Crivosin, PisaMéxico) por vía endovenosa lenta, en forma semanal, hasta que clínica y citológicamente se deje de apreciar el TVTc (Scarpelli et al., 2010).

\section{Evaluación Clínica}

Se realizó un examen físico general para determinar el grado de invasión, infecciones y condición corporal. Se registraron las alteraciones reportadas por los propietarios, tales como vómito, diarrea, depresión, dolor abdominal, y por el médico veterinario como fiebre, tamaño del tumor, presencia de secreciones, etc. (Scarpelli et al., 2010). Se consideró como «día 0 » el día que fueron diagnosticados con TVT. A partir de ese momento, se realizaron muestreos (hemograma y citología) semanales, antes de aplicar la quimioterapia.

Los pacientes fueron dados de alta al momento de la remisión del tumor. Se estimó que con pacientes que no se recuperasen después de seis aplicaciones de sulfato de vincristina, se recurriría al rescate con la combinación con doxorrubicina a las dosis recomendadas (Nak et al., 2005).

Se hizo una evaluación al finalizar el tratamiento para una revaloración de la correcta eliminación del TVTc y no reincidencia del mismo. Para esto, se tomaron muestras para citología clínica y PCR. Para las citologías, se localizó el área de lesión, realizando un lavado con solución de $\mathrm{NaCl} 0.9 \%$, se tomó la muestra con un hisopo y se hizo una extensión sobre un portaobjetos, el cual se tiñó con un hemocolorante. El material para PCR se tomó en forma similar y el hisopo se colocó en un tubo vacutainer con solución $\mathrm{NaCl} 0.9 \%$ y guardado en congelación hasta su procesamiento (extracción y PCR).

\section{Hematología y Citología}

Estudios de laboratorio (hemograma y citología) se hicieron en forma semanal antes del tratamiento para evaluar los efectos de la vincristina, de modo que si se detectaban datos de intoxicación (leucopenia, neutropenia, trombocitopenia o anemia), se suspendía temporalmente el medicamento hasta recuperar valores normales y reanudar la administración. En el caso de citología, se realizó para corroborar la presencia o ausencia de células neoplásicas, de modo que la ausencia de estas células en dos citologías consecutivas daba por concluido el tratamiento (Nak et al., 2005). 


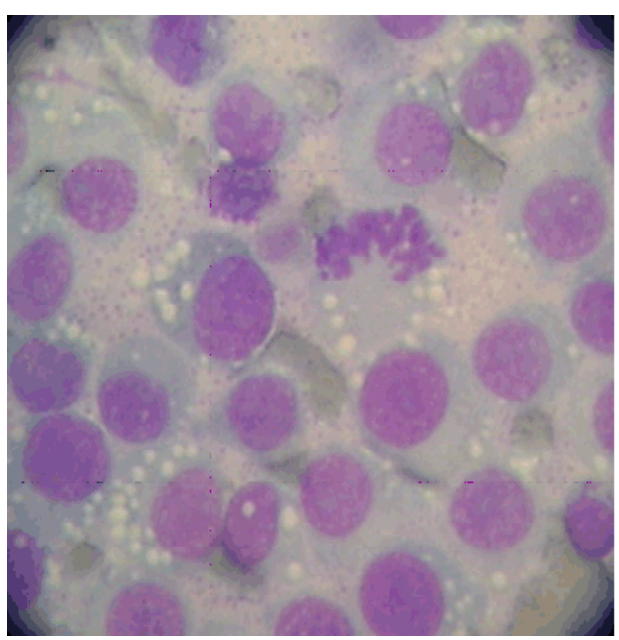

Figura 2. Citología de nódulo peneano de un perro, donde se observa una población pleomórfica de células redondas con moderado citoplasma, el cual era generalmente vacuolado y basófilo. Las características nucleares indican una neoplasia de células redondas con mitosis atípicas. Imagen compatible con TVTc, confirmado por PCR. 400x, Hemocolorante rápido Hycel

\section{Análisis Estadístico}

Se utilizó la prueba de «t» Student para comparar cambios en los valores hematológicos (volumen hematocrito, conteo plaquetario, actividad enzimática). Se utilizó la prueba exacta de Fisher para determinar diferencias entre el tipo de vincristina utilizada y el desarrollo de efectos adversos durante la quimioterapia y su asociación con la edad y sexo del perro. Para comparar el tiempo de respuesta al tratamiento se utilizó la prueba Log Rank-Test. Se usó el programa GraphPad Prism v. 6.0c (EEUU) para realizar los análisis estadísticos y las gráficas.

\section{Resultados}

El TVTc fue comprobado en 12 de los 13 perros del estudio mediante citología clínica y confirmado por PCR (figuras 1 y 2). Diez de ellos tuvieron una presentación geni-

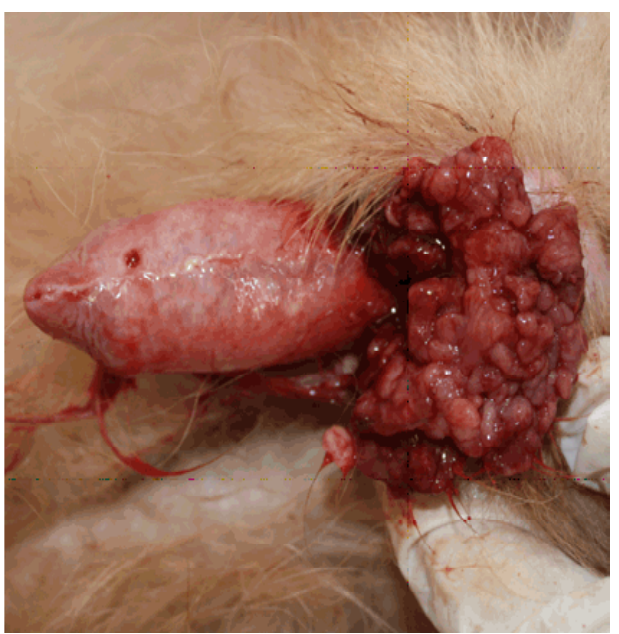

Figura 3. Típico nódulo multilobulado sangrante en la base de pene de un perro, con diagnóstico citológico y por PCR de tumor venéreo transmisible canino (TVTc)

tal (Fig. 3) y en los otros dos fue extragenital (cavidad nasal y subcutánea). Los resultados hematológicos y bioquímicos iniciales no mostraron cambios de importancia clínica en ambos grupos.

En los grupos Patente y Genérico se aplicó un máximo de cinco dosis para dar por concluido el tratamiento y el alta a los perros. El mínimo de aplicaciones en el grupo Patente fue de cuatro, mientras que en el grupo Genérico fue de tres aplicaciones; sin embargo, no se apreció diferencia significativa ni en el número de dosis aplicadas $(\mathrm{p}>0.05$, Wilcoxon) ni en el tiempo de remisión ( $p>0.05$, Log Rank Test).

El promedio de los resultados de los distintos analitos (hematocrito, plaquetas, leucocitos totales, neutrófilos) del hemograma no mostraron cambios significativos con respecto a los muestreos semanales ni entre grupos (Patente y Genérico); sin embargo, en el 
Cuadro 1. Cambios hematológicos (promedio \pm desviación estándar) en 6 perros con tumor venéreo transmisible canino (TVTc) tratados con $0.025 \mathrm{mg} / \mathrm{kg}$ de sulfato de vincristina genérica

\begin{tabular}{lccccccc}
\hline & \multicolumn{7}{c}{ Días de iniciada la terapia } \\
\cline { 2 - 8 } & 0 & 7 & 14 & 21 & $28^{1}$ & $35^{1}$ & $\begin{array}{c}\text { Valores de } \\
\text { referencia }^{2}\end{array}$ \\
\hline Hematocrito & $0.5^{\mathrm{a}} \pm 0$ & $0.5^{\mathrm{a}} \pm 0$ & $0.5^{\mathrm{a}} \pm 0$ & $0.5^{\mathrm{a}} \pm 0$ & $0.5^{\mathrm{a}} \pm 0$ & $0.5^{\mathrm{a}} \pm 0$ & $0.37-0.54$ \\
Plaquetas & $229^{\mathrm{a}} \pm 68$ & $334^{\mathrm{a}} 103$ & $229^{\mathrm{a}} \pm 61$ & $284^{\mathrm{a}} \pm 75$ & $392^{\mathrm{a}} \pm 112$ & $160^{\mathrm{a}} \pm 0$ & $200-900$ \\
Leucocitos & $12.5^{\mathrm{a}} \pm 3.2$ & $7.3^{\mathrm{b}} \pm 2.0$ & $8.8^{\mathrm{b}} \pm 4.3$ & $8.4^{\mathrm{b}} \pm 3.2$ & $7.5^{\mathrm{b}} \pm 1.9$ & $6.3^{\mathrm{b}} \pm 1.6$ & $6.0-17.0$ \\
Eosinófilos & $0.8 \pm 0.6$ & $0.3 \pm 0.2$ & $0.6 \pm 0.3$ & $0.6 \pm 0.4$ & $0.3 \pm 0.2$ & $0.1 \pm 0.0$ & $0.1-1.35$ \\
Neutrófilos & $10.3^{\mathrm{a}} \pm 3.0$ & $5.5^{\mathrm{b}} \pm 0.9$ & $6.4^{\mathrm{b}} \pm 3.3$ & $6.2^{\mathrm{b}} \pm 2.6$ & $5.1^{\mathrm{b}} \pm 1.1$ & $4.3^{\mathrm{b}} \pm 1.0$ & $3.0-11.5$ \\
segmentados & & & & & & &
\end{tabular}

${ }^{1} 5$ y 3 perros en los días 28 y 35 , respectivamente

2 Fuente: Valores del Hospital Veterinario para Pequeñas Especies, FM VZ-UAEM

${ }^{a, b}$ Superíndices diferentes dentro de filasindican diferencia significativa entre fechas $(p<0.05)$

Cuadro 2. Día y tipo de presentación de efectos adversos a la quimioterapia con sulfato de vincristina en perros con tumor venéreo transmisible canino (TVTc)

\begin{tabular}{cccc}
\hline Pacientes $^{1}$ & \multicolumn{3}{c}{ Días de presentación } \\
\hline P 1 & Vómitos & Diarreas & Mielosupresión \\
\hline P 2 & & & \\
P 3 & & & 28 \\
P 4 & 12 & 17 \\
P 5 & & 1 & 21 \\
P 6 & 10,12 & & 14 \\
G 1 & & & 21 \\
G 2 & & & 28 \\
G 3 & 7 & 13 & 7,14 \\
G 4 & 11 & & 7 \\
G 5 & 13 & & 35 \\
G 6 & 30 & & \\
\hline
\end{tabular}

${ }^{1}$ P: Vincristina de patente; G: Vincristina genérica

grupo Genérico se observó una disminución en el conteo promedio de leucocitos y neutrófilos del día 0 contra el resto de los muestreos $(\mathrm{p}<0.05)$ (Cuadro 1).
En ambos grupos se presentaron distintas reacciones adversas durante el tratamiento, las cuales fueron vómitos y diarrea, así como mielosupresión (Cuadro 2). En el gru- 
po Patente se presentaron cuatro pacientes (67\%) con este tipo de reacciones, especialmente vómitos, en comparación con el grupo Genérico donde todos los perros desarrollaron al menos una reacción adversa; sin embargo, no hubo diferencias estadísticas entre grupos ( $\mathrm{p}>0.05$, PE Fisher).

La mielosupresión fue el efecto adverso $(83 \%)$ más frecuente en ambos grupos, observándose en cuatro pacientes del grupo Patente y en los seis del grupo Genérico (Cuadro 2), diferencia que no llegó a ser estadísticamente significativa $(\mathrm{p}>0.05, \mathrm{PE}$ Fisher).

Siete perros ( 2 del grupo Patente y 5 del grupo Genérico) regresaron a la clínica un mes después de la finalización del tratamiento para confirmar vía PCR y citología clínica la ausencia del proceso patológico. Estos perros resultaron negativos a ambas pruebas, lo cual podría indicar que la remisión fue completa para ambos grupos.

\section{Discusión}

El diagnóstico clínico presuntivo del TVT está orientado por la morfología macroscópica y la localización; sin embargo, el diagnóstico definitivo se realiza por la citología clínica, biopsia (histopatología) y PCR (Mendoza et al., 2010; Ramírez et al., 2011). La citología es una prueba de diagnóstico rápido; sin embargo, la prueba de PCR es preferible por su mayor sensibilidad. En el presente estudio se tuvo un caso compatible con TVTc en el diagnóstico citológico pero negativo al PCR, por lo que fue excluido del estudio, y que en la evaluación histopatológica resultó ser un melanosarcoma amelánico. Es así que es probable que reportes de TVTc diagnosticados clínicamente y reportados como resistentes a la quimioterapia sean en realidad otro tipo de neoplasia $y$, por tanto, la respuesta a la terapia sea diferente. Los protocolos descritos tampoco indican el seguimiento citológico y hematológico de forma expresa y es probable que esto complique la determinación de la resolución clínica.

La edad, sexo y tipo de raza de los perros incluidos en este estudio no fue diferente a reportes previos como el de Das y Kumar (2000), quienes mencionan que perros de cualquier edad y sexo son susceptibles al TVTc; sin embargo, perros mayores de un año y de talla grande son más propensos, quizás por tener un mayor acceso a la calle, donde entran en contacto con perros vagabundos infectados.

La vincristina como agente único a dosis semanal de $0.025 \mathrm{mg} / \mathrm{kg}$ ha dado buenos resultados y con efectos colaterales mínimos (Vermooten, 1987; D’Amico et al., 2001). El pronóstico para la remisión total es favorable, siendo la administración de vincristina el tratamiento de elección. Un estudio controlado mostró que además del efecto citotóxico directo, los perros tratados con vincristina desarrollaron una mayor infiltración de linfocitos $\mathrm{CD}^{+}$(Gonzalez et al., 2000). En el presente estudio, la tasa de remisión clínica posterior a la quimioterapia fue del $100 \%$, sin aparente reincidencia hasta un mes de finalizado el tratamiento. No se observaron diferencias entre el tipo de medicamento empleado, indicando que tanto la presentación genérica como la de patente son igualmente eficaces para remitir el TVTc. No obstante, en el estudio de Nak et al. (2005), se requirió hasta siete aplicaciones de vincristina para eliminar el TVTc, pero, en ese caso, no mostraron el seguimiento clínico para determinar la remisión. En el presente estudio, la citología clínica fue de invaluable ayuda para determinar la ausencia de células de TVTc.

La vincristina tiene un efecto mielosupresor leve (Plum, 2006) y efectos secundarios como anorexia, vómito, diarrea y neutropenia (Tomiyasu et al., 2011). Sin embargo, pese a los problemas digestivos y mielosupresión observados en el presente estudio, estas manifestaciones no fueron de gran relevancia ya que los pacientes nunca 
presentaron una merma en su condición corporal durante el tratamiento. Asimismo, si bien en ambos grupos hubo mielosupresión, el promedio de neutrófilos en sangre fue la razón de la disminución de los conteos de leucocitos en el grupo genérico, por lo que se puede considerar que es la evaluación diferencial de neutrófilos, y no el conteo total de leucocitos, el parámetro para definir si debe suspenderse o no un protocolo quimioterapéutico para el tratamiento del TVTc.

En conclusión, los datos sugieren que tanto las presentaciones comerciales genéricas y de patente son apropiadas para el tratamiento del TVTc, ya que ambos tipos de medicamentos lograron una remisión total de los tumores. Asimismo, el desarrollo de reacciones adversas como la mielosupresión y alteraciones gastrointestinales son eventos a esperar, aunque siempre son ligeros $y$ autolimitantes.

\section{Literatura Citada}

1. Bautista-Gómez LG, MartínezCastañeda S, Córdova-Alarcón E, Vásquez-Chagoyán JC. 2011. Analysis of canine transmissible veneral tumor genotypes using the D-loop region of mitochondrial DNA. Genes Genet Syst. 86: 351-355. doi: 10.1266/ggs.86.351

2. Brown NO, Calvert C, MacEwen G. 1980. Chemotherapeutic management of transmissible venereal tumors 30 in dogs. J Am Vet Med Assoc 176: 983986.

3. D'Amico G, Adagio L, Lattanzi D, Álvarez A. 2001. Tumor venéreo transmisible: importancia del tratamiento quimioterápico. En: Argentina: Anuario Facultad de Ciencias Veterinarias, Universidad Nacional de La Pampa. p 34-39.

4. Das U, Kumar A. 2000. Review of canine transmissible venereal sarcoma. Vet Res Commun 24: 545-556.

5. Erünal-Maral N, Findik M, Aslan $S$. 2000. Use of exfoliative cytology for diagnosis of transmissible venereal tumor and controlling the recovery period in the bitch. Dtsch Tierarztl Wochenschr 107: 175-180.

6. Gonzalez C, Griffey S, Naydan D, Flores $E$, Cepeda $G$, Cattaneo $G$, Madewell B. 2000. Canine transmissible venereal tumor: a morphological and immunohistochemical study of 11 tumors in growth phase and during regression after chemotherapy. J Comp Pathol 122: 241-248. doi: 10.1053/jcpa.1999.0366

7. Grau-Bassas E, Kociba G, Couto C. 2000. Vincristine impairs platelet aggregation in dogs with lymphoma. J Vet Intern Med 14: 81-85. doi: 10.1111/ j.1939-1676.2000.tb01503.x

8. Hsiao Y, Liao K, Hung S, Chu R. 2002. Effect of tumor infiltrating lymphocytes on the expression of MHC molecules in canine transmissible venereal tumor cells. Vet Immunol Immunopathol 87: 19-27. doi: 10.1016/S0165-2427(02)00026-0

9. Liao KW, Lin ZY, Pao HN, Kam SY, Wang FI, Chu RM. 2003. Identification of canine transmissible venereal tumor cells using in situ polymerase chain reaction and the stable sequence of the long interspersed nuclear element. J Vet Diagn Invest 15: 399-406.

10. Marcos R, Santos M, Marrinhas $C$, Rocha E. 2006. Cutaneous transmissible venereal tumor without genital involvement in a prepubertal female dog. Vet Clin Pathol 35: 106-109.

11. Mendoza N, Chavera A, Falcón N, Perales $R$. 2010. Frecuencia del tumor venéreo transmisible en caninos: casuística del Laboratorio de Patología Veterinaria de la Universidad Nacional Mayor de San Marcos (periodo 1998 - 2004). Rev Inv Vet Perú 21: 32-47. doi: 10.15381/ rivep.v21i1.305

12. Mukaratirwa S, Gruys E. 2003. Canine transmissible venereal tumor: cytogenetic origin, immunophenotype and immuno-biology. A review. Vet Q 25: 3: 101-111. doi: 10.1080/01652176.2003.9695151 
13. Murgia C, Pritchard J, Kim S, Fassati A, Weiss R. 2006. Clonal origin and evolution of a transmissible cancer. Cell 126: 477-487. doi: 10.1016/j.cell.2006.05.051

14. Nak D, Nak Y, Cangul T, Tuna B. 2005. A clinico-pathological study on the effect of vincristine on transmissible venereal tumor in dogs. J Vet Med A Physiol Pathol Clin Med 52: 366-370.

15. Ortega PA, Acevedo AM, Sauri AC, Bolio GM, Gutiérrez BE. 2003. Prevalencia de tumor venéreo transmisible en perros callejeros de la ciudad de Mérida, Yucatán, México. Rev Biomed 14: 8387.

16. Plumb D. 2006. Manual de farmacología veterinaria. $5^{\circ}$ ed. Buenos Aires: Inter-Médica. $1256 \mathrm{p}$.

17. Ramírez YE, Reyes AIR, Rodríguez Y, Cuesta GAF, Pérez CLE. 2011. Tumor venéreo transmisible en un perro mestizo y su respuesta a la quimioterapia citostática. Redvet 12(6). [Internet]. Disponible en: http://www.veterinaria.org/revistas/redvet/n060611/061102.pdf

18. Salamanca S, Santader-Baquero A, Triana-García PA, Romero $S$, Rondón-Barragán IS. 2008. Tumor venéreo transmisible (TVT) con metástasis pulmonar: reporte de caso. Orinoquia 12: 162-170.
19. Scarpelli K, Valladäo M, Metze K. 2008. Predictive factors for the regression of canine transmissible venereal tumor during vincristine therapy. Vet J 183: 362-363. doi: 10.1016/ j.tvjl.2008.11.009

20. Sorenmo K, Hardwood L, King L, Drobatz K. 2010. Case-control study to evaluate risk factors for the development of sepsis (neutropenia and fever) in dogs receiving chemotherapy. J Am Vet Med Assoc 236: 650-656. doi: 10.2460/ javma.236.6.650

21. Strakova A, Murchison E. 2014. The changing global distribution and prevalence of canine transmisible venereal tumour. BMC Vet Res 10: 168. doi:10.1186/s12917-014-0168-9

22. Tomiyasu H, Takahashi M, Fujino Y, Ohno K, Tsujimoto H. 2011. Gastrointestinal and hematologic adverse events after administration of vincristine, cyclophosphamide, and doxorrubicin in dogs with lymphoma that underwent a combination multidrug chemotherapy protocol. J Vet Med Sci 72: 1391-1397.

23. Vermooten M. 1987. Canine transmissible venereal tumor (TVT): a review. J S Afr Vet Assoc 58: 147-150.

24. VonHoldt B, Ostrander E. 2006. The singular history of canine transmissible tumor. Cell 126: 445-447. doi: 10.1016/ j.cell.2006.07.016 